\title{
Um Assistente de Recomendação Sensível ao Contexto para Ambientes Virtuais de Aprendizagem baseados na Metodologia da Problematização
}

\author{
Fausto J. F. B. Gominho ${ }^{1}$, Patrícia Tedesco ${ }^{1}$, Rosalie B. Belian ${ }^{2}$ \\ ${ }^{1}$ Centro de Informática \\ Universidade Federal de Pernambuco (UFPE) - Recife - PE \\ ${ }^{2}$ Centro de Ciências da Saúde \\ Universidade Federal de Pernambuco (UFPE) - Recife - PE
}

fjfbg@cin.ufpe.br, pcart@cin.ufpe.br, rosalie.belian@gmail.com

\begin{abstract}
Resumo. A utilização da metodologia da problematização em ambientes aprendizagem apoiados por tecnologia tem a vantagem de estimular o aprendizado ativo do aprendiz, promovendo a sua autonomia no processo de construção do conhecimento. Afim de auxiliar esse processo, este trabalho apresenta um assistente de recomendação de recursos de aprendizagem sensivel ao contexto para ambientes virtuais de aprendizagem baseado na Metodologia da Problematização operacionalizada através do Arco de Charles Maguerez.
\end{abstract}

\begin{abstract}
Using the methodology of problematization in technology-enhanced learning has the advantage of stimulating active learning of the learner, promoting their autonomy in the process of knowledge construction. In order to assist this process, this paper presents a context-aware recommendation assistant of learning resources for virtual learning environments based on the Methodology of Problematization operationalized through the Arch of Charles Maguerez.
\end{abstract}

\section{Introdução}

No modelo de ensino tradicional, o aprendiz é tratado como um simples receptor e armazenador de informações, sendo que muitas destas informações não são associadas à realidade e experiência prática do aprendiz (GOMES, 2014). Diante deste cenário, esse modelo de ensino aos poucos vem sendo substituído por novas tendências pedagógicas, as quais apontam para a necessidade da formação de um profissional capaz de transformar a realidade social do seu cotidiano (PRADO et al., 2012). A Metodologia da Problematização tem como primeira referência o Método do Arco, de Charles Maguerez e de acordo com a sua proposta, é possível aprender determinado tema quando se adotam as seguintes etapas: observação da realidade; identificação dos pontos-chaves, teorização, desenvolvimento de hipótese de solução e aplicação à realidade prática (BERBEL, 1998).

A utilização da metodologia da Problematização em um ambiente virtual de aprendizagem (AVA) tem a vantagem de estimular o aprendizado ativo do estudante, 
promovendo a sua autonomia no processo de construção de conhecimento (GOMES, 2014). Nesse processo autônomo de construção do conhecimento, o aluno se torna responsável por pesquisar conteúdos e utilizar ferramentas que o auxiliem na resolução do problema. Apesar desses conteúdos estarem disponíveis em diversos repositórios virtuais, a grande quantidade de recursos acessíveis pode ocasionar uma sobrecarga de informação no aluno. Sendo que o ideal seria que os sistemas percebessem a necessidade do usuário e, com isso, minimizassem a complexidade e esforço destes na busca por solução (VIEIRA; TEDESCO; SALGADO, 2009). Desta forma, este artigo apresenta a construção de assistentes de recomendação de recursos de aprendizagem integrado a um ambiente baseado na Metodologia da Problematização e como o contexto pode ser integrado a este assistente.

Este artigo está dividido em 4 seções, onde a seção 2 mostra uma visão geral sobre sistemas de recomendação no âmbito educacional e a utilização de contexto computacional para prover conteúdos adequados aos aprendizes. A seção 3 descreve a abordagem proposta neste artigo. Já a última seção apresenta as considerações finais.

\section{Sistemas de Recomendação Educacional Sensíveis ao Contexto}

Diante da grande quantidade de informação disponibilizada através da internet e da facilidade de acesso a estas informações, os sistemas de recomendação têm como objetivo o desenvolvimento de aplicações que ajudem no processo de recomendação de conteúdos e serviços personalizados e que sejam úteis para o usuário, diminuindo assim o a sua sobrecarga cognitiva (ADOMAVICIUS; TUZHILIN, 2005). Para CAZELLA et al., (2012), os Sistemas de Recomendação utilizam repositórios de informação e dados do perfil do usuário, para auxilia-lo no processo de seleção de conteúdo de maneira que os conteúdos selecionados atendam as expectativas deles.

A aprendizagem apoiada por tecnologia (technology enhanced learning, TEL) tem como objetivo definir, desenvolver e testar inovações sócio técnicas que melhorem as práticas de aprendizagem tanto individualmente como em organizações (MANOUSELIS et al., 2011). Neste contexto, os sistemas de recomendação têm o objetivo de recomendar recursos de aprendizagem adequados a um determinado aluno ou grupo de alunos. Para isso os sistemas precisam funcionar integrados a uma infraestrutura de e-learning de modo a ser capaz de receber e fornecer as informações necessárias ao processo de recomendação (DRACHSLER; HUMMEL; KOPER, 2008).

A incorporação de informações contextuais dos aprendizes no processo de recomendação é importante para adequação das recomendações de acordo com as características de cada indivíduo, tais com o tempo disponível, localização atual, pessoas próximas e etc (VERBERT et al., 2012). Apesar de existirem várias definições de contexto, neste trabalho utilizamos a definição de VIEIRA, (2008), na qual faz uma distinção entre os conceitos de contexto e de elementos contextuais e os definiu da seguinte maneira: Um elemento contextual é qualquer dado, informação ou conhecimento que permite caracterizar uma entidade em um domínio, e o contexto é o conjunto de elementos contextuais instanciados que são necessários para apoiar a execução de alguma tarefa.

Alguns trabalhos foram usados como referência para este artigo, por usarem sistemas de recomendação no domínio da educação (FERREIRA, GLUZ, BARBOSA, 
2012; GÓMEZ et al., 2014; REZENDE et al., 2013; SILVA, NETO, JÁCOME JR, 2011; ZAINA et al., 2012).

\section{Um Assistente de Recomendação baseado na Metodologia da Problematização}

Este trabalho propõe um assistente de recomendação sensível ao contexto integrado a um ambiente educacional baseado na Metodologia da Problematização e operacionalizado pelo Arco de Maguerez. Para isso foi utilizado o ambiente educacional PenSAE, proposto por GOMES, (2014), que utiliza o método do Arco de Maguerez com foco na aquisição de competências e habilidades necessárias à prática do processo de Enfermagem. A utilização do PenSAE como base para o projeto se deu pela verificação que o mesmo poderia ser utilizado em processos de problematização em qualquer área de conhecimento, necessitando de apenas algumas adaptações.

Uma instância do assistente de recomendação integrado ao ambiente de aprendizagem foi desenvolvida, e denominada PenSAE+. Para projetar o assistente, foi utilizada a documentação existente do PenSAE como entrada para o framework CEManTIKA (VIEIRA, 2008). O framework tem como objetivo apoiar o projeto de sistemas sensíveis ao contexto em diferentes domínios e é composto por quatro elementos principais: uma arquitetura genérica para sistemas sensíveis ao contexto, um metamodelo de contexto independente de domínio, um conjunto de perfis UML (Unified Modeling Language) e um processo que direciona a execução de atividades relacionadas à especificação do contexto e ao projeto de sistemas sensíveis ao contexto. O protótipo foi desenvolvido em Ruby on Rails e banco de dados PostgreSQL e cobriu as funcionalidades relacionadas a resolução de estudo de caso, percorrendo as cinco etapas do Arco de Maguerez. O assistente foi implementado para realizar recomendação de recursos de aprendizagem (objetos de aprendizagem, artigos e livros) na fase de teorização, conforme figura 1 .

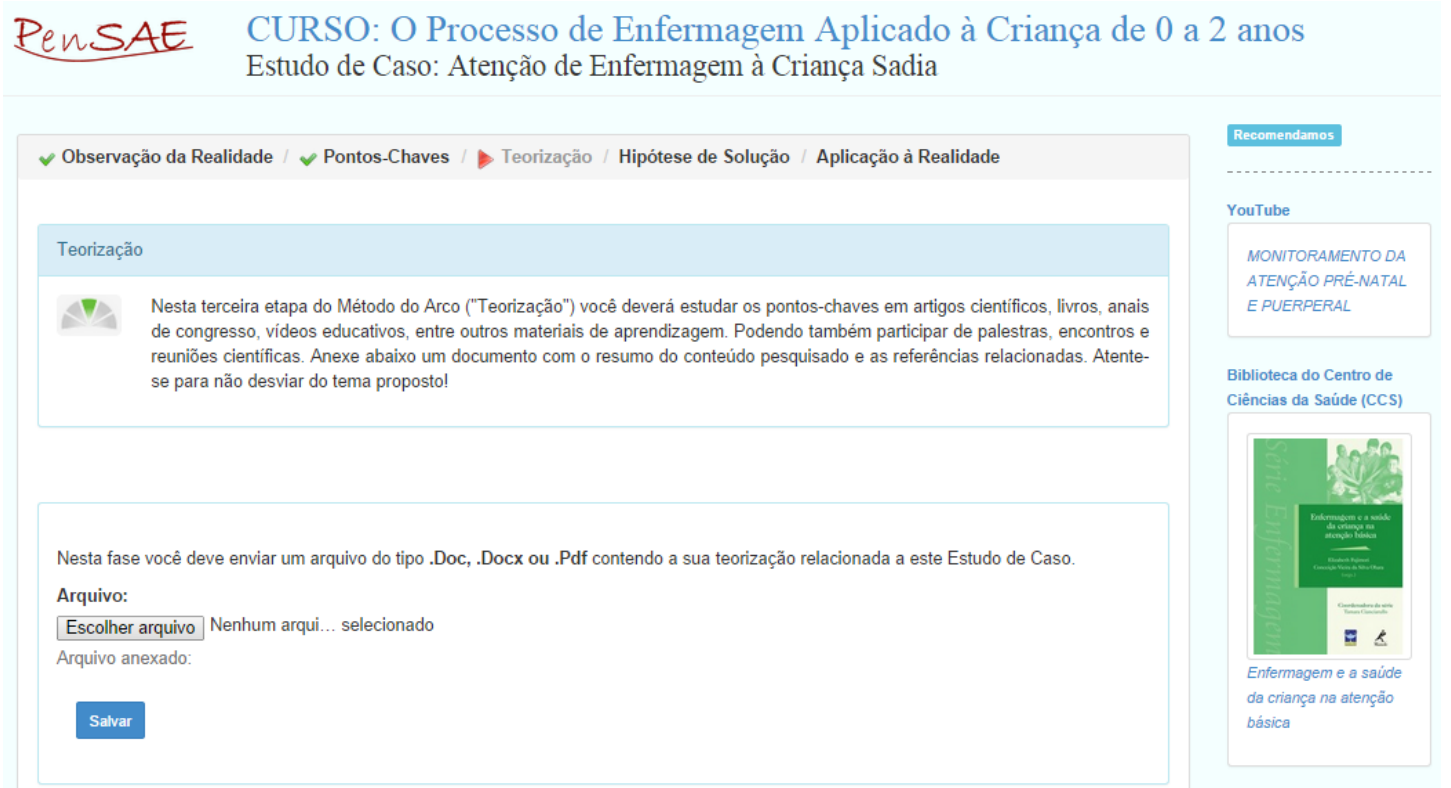

Figura 1. Recomendações do Assistente na fase de Teorização do Arco 
No protótipo desenvolvido, as recomendações se baseiam nas preferências do tipo de material no qual o aluno prefere estudar e no domínio do estudante nos idiomas português, inglês e espanhol.

\section{Considerações Finais}

A utilização da tecnologia da informação e comunicação no domínio da educação e os avanços da internet, vem disseminado a utilização dos AVAs. Nestes ambientes a tecnologia é emprega para dar suporte as atividades educacionais, fornecendo ferramentas que facilitam a aprendizagem dos alunos. Os ambientes educativos que utilizam como método de ensino a problematização, têm a vantagem de estimular o aprendizado ativo do estudante, promovendo a sua autonomia no processo de construção do conhecimento. Com o propósito de auxiliar o aluno no uso eficiente dos recursos disponíveis em AVAs, surgem os sistemas de recomendação educacionais.

Os trabalhos apresentados têm focado na recomendação de recursos sem a preocupação com a metodologia de ensino empregada no processo de aprendizagem. Neste trabalho apresentamos uma abordagem para recomendação de recursos de aprendizagem sensível ao contexto do aluno e baseada na metodologia da Problematização.

\section{References}

ADOMAVICIUS, G.; TUZHILIN, A. Towards the Next Generation of Recommender Systems: A Survey of the State-of-the-Art and Possible Extensions. IEEE Transactions on Knowledge and Data Engineering, p. 734-749, 2005.

BERBEL, N. A. N. A problematização ea aprendizagem baseada em problemas. Interface Comun Saúde Educ, v. 2, n. 2, p. 139-154, 1998.

CAZELLA, S. C. et al. Desenvolvendo um Sistema de Recomendação de Objetos de Aprendizagem baseado em Competências para a Educação: relato de experiênciasAnais do Simpósio Brasileiro de Informática na Educação. Anais...2012

DRACHSLER, H.; HUMMEL, H. G.; KOPER, R. Personal recommender systems for learners in lifelong learning networks: the requirements, techniques and model. International Journal of Learning Technology, v. 3, n. 4, p. 404-423, 2008.

FERREIRA, L. G. A.; GLUZ, J. C.; BARBOSA, J. L. V. Um Modelo Multiagente para Recomendação de Conteúdo Educacional em um Ambiente UbıquoAnais do Simpósio Brasileiro de Informática na Educação. Anais...2012

GOMES, R. L. V. APLICAÇÃO DE UMA ESTRATÉGIA DE ENSINO PROBLEMATIZADORA NA ASSISTÊNCIA DE ENFERMAGEM À CRIANÇA DE ZERO A DOIS ANOS: SUBSÍDIOS PARA O PROCESSO DE ENSINOAPRENDIZAGEM. 2014. 190 f. Tese (Doutor em Saúde da Criança e do Adolescente) - Centro de Ciências da Saúde, Universidade Federal de Pernambuco, Recife, 2014. 
GÓMEZ, S. et al. Context-aware adaptive and personalized mobile learning delivery supported by UoLmP. Journal of King Saud University-Computer and Information Sciences, v. 26, n. 1, p. 47-61, 2014.

MANOUSELIS, N. et al. Recommender Systems in Technology Enhanced Learning. In: Recommender Systems Handbook. 2011. Springer, 2011. p. 387-409.

PRADO, M. L. DO et al. Arco de Charles Maguerez: refletindo estratégias de metodologia ativa na formação de profissionais de saúde. Esc. Anna Nery Rev. Enferm, v. 16, n. 1, p. 172-177, 2012.

REZENDE, P. et al. BROAD-RS: UMA ARQUITETURA PARA RECOMENDAÇÃO DE OBJETOS DE APRENDIZAGEM SENSÍVEL AO CONTEXTO USANDO AGENTES. 2013.

SILVA, L. C. N. DA; NETO, F. M. M.; JÁCOME JR, L. MobiLE: Um ambiente Multiagente de Aprendizagem Móvel para Apoiar a Recomendação Sensıvel ao Contexto de Objetos de Aprendizagem. Anais do XXII SBIE - XVII WIE, 2011.

VERBERT, K. et al. Context-aware recommender systems for learning: a survey and future challenges. Learning Technologies, IEEE Transactions on, v. 5, n. 4, p. 318$335,2012$.

VIEIRA, V. CEManTIKA: A domain-independent framework for designing context-sensitive systems. 2008. 187 f. Tese (Doutorado) - Universidade Federal de Pernambuco, Recife, 2008.

VIEIRA, V.; TEDESCO, P.; SALGADO, A. C. Modelos e Processos para o desenvolvimento de Sistemas Sensıveis ao Contexto. André Ponce de Leon F. de Carvalho, Tomasz Kowaltowski.(Org.). Jornadas de Atualização em Informática, p. 381-431, 2009.

ZAINA, L. A. et al. e-lors: Uma abordagem para recomendacao de objetos de aprendizagem. Revista Brasileira de Informática na Educação, v. 20, n. 1, p. 04, 2012. 